\title{
IdeAs
}

Idées d'Amériques

$4 \mid 2013$

Crises et effets de crise dans les Amériques

\section{Les crises aux Etats-Unis depuis 1837 : facteurs de renouveau et de redéploiement}

Crises in the US since 1837 as Generating New Forms of Deployment and

Development

Crisis en los Estados Unidos desde 1837 : fuentes de nuevas formas de extensión y expansión

\section{Jean Luc Tendil}

\section{OpenEdition}

\section{Journals}

Édition électronique

URL : https://journals.openedition.org/ideas/685

DOI : $10.4000 /$ ideas.685

ISSN : 1950-5701

Éditeur

Institut des Amériques

Référence électronique

Jean Luc Tendil, « Les crises aux Etats-Unis depuis 1837 : facteurs de renouveau et de

redéploiement », IdeAs [En ligne], 4 | 2013, mis en ligne le 15 octobre 2013, consulté le 18 octobre

2022. URL : http://journals.openedition.org/ideas/685 ; DOI : https://doi.org/10.4000/ideas.685

Ce document a été généré automatiquement le 18 octobre 2022.

\section{c) (i) $९$}

Creative Commons - Attribution - Pas d'Utilisation Commerciale - Pas de Modification 4.0 International - CC BY-NC-ND 4.0

https://creativecommons.org/licenses/by-nc-nd/4.0/ 


\title{
Les crises aux Etats-Unis depuis 1837 : facteurs de renouveau et de redéploiement
}

\author{
Crises in the US since 1837 as Generating New Forms of Deployment and
}

Development

Crisis en los Estados Unidos desde 1837 : fuentes de nuevas formas de extensión y expansión

Jean Luc Tendil

\section{Introduction}

2 Les crises économiques et morales, aux États-Unis, sont-elles consubstantielles à leur prospérité houleuse, insolente et imprévisible? Toujours est-il que les dépressions vertigineuses dans lesquelles ce pays a maintes fois été plongé n'ont d'égal que l'essor prodigieux de ses cités verticales. Aussi nous proposons-nous d'envisager la crise à la fois comme une défaillance morale intervenant avant la panique financière et une éventuelle prise de conscience qui lui serait subséquente.

3 Krei, en grec, désigne le discernement entre deux états du monde. La crise n'est pas seulement la zone dépressionnaire qui sépare deux périodes de croissance, c'est le moment où des hommes doués de discernement décident et tranchent, pour le pire ou pour le meilleur; c'est le moment pour l'Amérique pressée de prendre le temps du regard intérieur critique ${ }^{1}$ à la recherche de crimes $^{2}$ et de bienfaits.

4 Les paniques $\mathrm{du}$ XIX ${ }^{\text {ème }}$ siècle nous intéressent particulièrement parce qu'elles interviennent dans une Amérique en gestation, ivre de son devenir, et parce que les États-Unis ont construit leur imaginaire en grande partie autour des grandes crises de cette époque et des rebonds qui les ont suivies. Rebonds d'une extraordinaire 
agressivité et parfois ponctuées de découvertes fabuleuses - certains diraient providentielles - comme celles de l'or : quand le hasard met l'or, en temps de crise, sur le chemin du voyageur américain errant à la lisière du désespoir, il le conforte dans sa foi en la Providence.

Nous nous intéresserons aux crises de 1837 et de 1893, chacune suivie par un prurit expansionniste déterminant pour l'évolution des États-Unis.

6 Nous verrons ensuite comment l'or, découvert à la marge du territoire américain ${ }^{3}$, agit comme un dérivatif aux angoisses de la crise morale dans un pays où tout évènement historique se prête aux interprétations religieuses (l'or révèle l'homme ou la nation dans ce qu'ils ont de pire et de meilleur).

7 Enfin nous verrons en quoi ces crises sont le miroir prophétique de la crise actuelle qui démarre peut-être avant 2008 ; comment l'Homo Americanus, poussé dans la crise par une boulimie faustienne, réagit en sortant de lui-même, en agressant le monde ou en allant à sa rencontre.

\section{Le déroulement de deux grandes crises : 1837 et 1893}

8 La crise de 1837 intervient dans une jeune république qui surprend déjà le monde, Tocqueville en tête, par sa prospérité, sa hardiesse à la fois individualiste et patriotique, et son influence potentielle sur le cours de l'histoire mondiale.

9 Sous Andrew Jackson, héros de la conquête de l'ouest élu en 1829, la population américaine croît rapidement. Les plantations de tabac ou de coton du sud épuisent la terre et obligent les planteurs, toujours plus nombreux, à rechercher des terres encore vierges et fertiles plus à l'ouest. La création d'emplois dans le secteur manufacturier étant encore faible, à part en Nouvelle Angleterre, encore célèbre pour ses distilleries de rhum, l'ouest transappalachien est le débouché évident des immigrants ou des enfants d'immigrés. La séduction de l'ouest est d'autant plus forte qu'il répond au rêve jeffersonien d'exploitation de la terre par l'homme et pour l'homme, pensé comme un propriétaire revendiquant sa part d'oisiveté.

10 Jackson favorise l'installation des colons au-delà des Appalaches en signant l'acte de déportation des Indiens (1830), tache sombre dans l'histoire américaine, et en favorisant le développement des infrastructures.

11 Il confie également l'argent de la Banque des États-Unis à des banques locales (surnommées pet banks ou "banques choyées» en raison des faveurs douteuses dont elles bénéficièrent) afin de faciliter les prêts aux futurs exploitants des terres de l'ouest.

12 En fait, cet argent sert surtout les promoteurs qui revendent avec plus-value. Les prix sont rapidement et dangereusement déconnectés du potentiel productif et lucratif de la terre - d'autant plus que certaines cultures, comme le coton, sont déjà au bord de la surproduction.

13 Inquiet, Jackson décide peu avant la fin de son deuxième mandat que les terres fédérales, à l'ouest, seront dorénavant payées en espèces, or ou argent. Il précipite ainsi, à la fin de son mandat, la panique qui guettait:promoteurs et banques, incapables de produire les espèces désormais exigées, font faillite en cascade ; fermes, 
manufactures et simples boutiques suivent; le chômage s'envole sur l'ensemble du territoire. Selon l'historien Howard Zinn, un tiers des travailleurs de New York se retrouve sans travail en 1837. Deux cent mille - sur un total de cinq cent mille - sont dans la détresse (Zinn, 2002).

L'erreur de Jackson fut peut-être sa foi illimitée dans le pouvoir régénérateur de la frontière - il ne voyait plus que la terre, au-delà des transactions financières, devait produire; et que c'est la capacité d'absorption de ce qu'elle produit par les acteurs économiques qui déterminera la prospérité et la liberté de ses occupants. Autrement dit, il négligeait le travail de digestion qui accompagne un développement économique sain.

La frontière magique métamorphose l'individu et lui offre ainsi une métaphore autant qu'un prolongement de son affranchissement spirituel : don providentiel aux chrétiens espagnols libérés de l'Islam, puis, un siècle plus tard, aux Puritains fuyant l'Église anglicane, voilà comment l'Amérique fut d'abord perçue. Même la Virginie fut ce don de pureté à l'homme que l'Europe a sali.

16 Jackson, lui-même « conquistador » de l'ouest américain, contribua à faire de l'homme de la Frontière le détenteur d'une énergie surhumaine, celui qui fait du don de la terre l'instrument d'une puissance bienfaitrice et méritée. Là réside peut-être l'une des ambiguïtés fondatrices de l'Amérique et l'origine de ses crises:ce qu'elle aime chez l'homme, c'est le surhomme. Voilà pourquoi, comme le disait Jules Verne et le rappelait Paul Auster, «rien n'étonne un Américain $»^{4}$ - rien ne l'étonne de la part de l'Homme, puisque les surhommes sont dans la foule, et qu'au nom de tous ils conquièrent et façonnent le monde dans lequel nous évoluons.

17 Un tel enthousiasme, cependant, est plus faustien que chrétien:l'Amérique « théophile » devient momentanément « théophage » sous Jackson ; elle penche vers un égoïsme sans Dieu et sans dessein. La crise de 1837 est peut-être l'occasion d'un regard intérieur; non pas vraiment d'une remise en cause, mais d'une réintégration de l'Histoire américaine dans la ferveur retrouvée d'un récit messianique. L'Amérique de Van Buren, Harrison, Tyler et Polk, successeurs de Jackson, est plus que jamais conquérante, car toute crise appelle un redéploiement, mais elle donne à ses conquêtes barbares des accents mystiques et des teintes visionnaires. À la conquête factice et oublieuse de Dieu qui précède la crise succède une conquête véritable qui voit débusqués et vaincus les ennemis d'un Dieu allié de l'Amérique. Le journaliste John O'Sullivan invoque en 1845 la Providence et la «destinée manifeste» du peuple américain (O'Sullivan, 1845). La démesure en Dieu remplace la démesure sans Dieu. Le regard sur soi consécutif à la crise n'est pas forcément une prise de conscience, mais peut-être un nouvel aveuglement.

18 La destinée manifeste autorise l'annexion du Texas la même année, alors que les colons anglophones majoritaires et issus d'autres états américains, en rébellion contre le gouvernement mexicain, l'avaient déclaré provisoirement indépendant en 1836.

19 L'ardeur guerrière des Texans, puis du gouvernement américain, rachète peut-être l'Amérique de sa ruine morale et de sa démesure en consacrant une énergie héroïque à l'épopée nationale. Le poète Walt Whitman en donne un aperçu :

Oui le Mexique doit être sévèrement châtié. (...) Que nos armes soient désormais portées de manière à apprendre au monde entier que, bien que nous n'aimions pas les querelles, l'Amérique sait comment frapper et connaît les moyens de s'étendre. (Zinn H., 2002 : 181) 
On remarque l'allusion quasiment religieuse au châtiment. L'Amérique orgueilleuse s'immerge dans l'oubli de ses échecs et de ses failles et préfère désigner les vagues crimes d'un étranger méconnu. Elle n'est pas seulement purgée par son oubli volontaire, mais plus encore exaltée par la mission sacrée qu'elle accomplit dans l'agression - combien d'observateurs de cette époque n'ont-ils pas souligné les vices du peuple mexicain fantasmé avant d'avoir été approché ?

Le vague sentiment d'une faute nationale existe peut-être, mais il est minoritaire et atténué par la quasi-certitude que les fautes des autres sont rédhibitoires, quand les fautes dont on est soi-même coupable sont vénales, voire justifiables ${ }^{5}$.

L'introspection fait peur, et ce qu'on y cherche est moins la raison que la voix martiale d'un Dieu qui avance. Loin d'enseigner la mesure et la sobriété, la crise libère les désirs et la fièvre intérieure de ceux qui la subissent ou la perçoivent. Elle met l'Homo Americanus face à ce qu'il considère comme la plus grande menace envers son existence, et peut-être le plus grand péché de l'homme envers l'homme:la paresse et l'immobilisme sociaux (ceux qu'on prête au Mexique, par exemple). La vie du chrétien, en effet, telle qu'elle est conçue par John Bunyan, ce puritain anglais qui inspira l'Amérique à la fin du XVII ${ }^{\text {ème }}$ siècle, est une pérégrination préalable à l'existence posthume en Dieu. La guerre est toujours le remède le plus facile contre la sclérose coupable qui guette et qui gagne, et tant pis si on s'y enivre d'une violence qui oublie sa fin. C'est encore Zinn qui rapporte un témoignage illustrant cette réaction irrationnelle contre un sentiment d'immobilisme semblant frapper l'Amérique à cette époque :

Partons en guerre. Le monde est devenu triste et insipide, tous les navires doivent être capturés et les cités abattues. Et le globe mis à feu et à sang afin que nous puissions redémarrer ${ }^{6}$. Ce pourrait être drôle. Quelque chose d'intéressant. Dont on puisse parler. (Zinn H., 2002 : 185)

3 On voit ici se dessiner le lien avec la crise : il s'agit de redémarrer; c'est à dire de relancer l'industrie et le commerce, et par là même d'une réappropriation par l'Amérique de sa mission historique dévoyée. En somme, il s'agit bien d'une réaction à la crise économique et morale, ce moment de torpeur où l'on craint que tout ne finisse par s'arrêter.

4 Preuve que la réaction relève de l'élan messianique, l'Amérique lorgne alors sur la côte Pacifique. Ce n'est pas seulement un territoire que l'on désire, c'est le commerce mondial que l'on convoite, fût-il violent et sans pitié. En évoquant le Pacifique, on souffle sur les braises du vieux rêve des Indes. Si la conquête de l'ouest est une guerre sainte, l'océan offre la garantie de ne jamais s'arrêter en si bon chemin. Si la crise qui voit chuter le commerce international attise la peur de l'immobile, alors la perspective d'un commerce mondial régi et stimulé par l'Amérique permet peut-être de conjurer la peur d'un monde qui, ne commerçant plus, n'est plus capable de respirer ni de tournoyer dans une puissante unité organique. L'Amérique fait déjà le rêve fou de la mondialisation, d'autant plus passionnément qu'elle est déjà hantée par le cauchemar de sa propre mort, cernée par des océans qui pourraient redevenir hermétiques.

En 1845, le président James Polk, qui n'est peut-être pas un stratège, n'en fait pas moins du Pacifique une idée fixe ${ }^{7}$. Le soir même de son investiture, il confie à son secrétaire à la Marine que l'un de ses principaux objectifs est d'acquérir la Californie, alors possession mexicaine. Il fera donc tout pour titiller la susceptibilité de ce voisin troublé et provoquer ainsi un conflit lui permettant de s'emparer de la Californie, et de sa côte ouverte aux vents de l'Asie. Ayant signé l'acte d'intégration du Texas dans l'Union, il 
enverra ses troupes bousculer la trébuchante armée mexicaine à la frontière sud du nouvel état, obtiendra la guerre tant espérée, et la victoire qui relancera l'Amérique. La crise avait bien engendré son redéploiement, impitoyable à défaut d'être vraiment providentiel. La crise de 1893, nous allons le voir, fut similaire par le déroulement et par le dénouement.

La crise de 1893 intervient elle aussi dans une période de développement humain et économique consécutif à la guerre civile, période impatiente marquée par des investissements massifs dans le chemin de fer. Ces derniers doivent permettre aux fermiers et aux compagnies minières de l'ouest d'écouler leur production, et de relier entre elles les grandes agglomérations conçues comme des lieux d'échange dont l'irrigation favorise la prospérité de l'ensemble. La même obsession boulimique du quadrillage de l'espace américain qui avait caractérisé la crise de 1837 se fait jour. On ne veut plus voir que le chemin de fer doit s'adapter au rythme de la croissance démographique et commerciale. On préfère s'aveugler de la certitude qu'il crée luimême, et partout, les débouchés qui rendent son exploitation rentable.

À cette frénésie, et stimulée par elle, s'ajoute la surproduction minière, notamment celle des mines d'argent. Afin d'éviter l'effondrement des cours et la faillite des compagnies minières, le gouvernement achète l'argent en grande quantité pour frapper monnaie, inondant ainsi le marché de liquidités qui encouragent la spéculation. Les acteurs économiques, semble-t-il, n'acceptent plus la lenteur du temps économique, ne veulent plus prendre le temps de digérer cet espace énorme qu'est l'Amérique - ce moment de la digestion ressemble trop à l'immobilité du paresseux, au sommeil magique d'un Rip Van Winkle ${ }^{8}$ pendant lequel l'histoire et la terre qu'il croyait siennes échappent à son emprise fragile.

Surcapacités et spéculation fiévreuse provoquent ainsi la crise de 1893. Banques et compagnies minières en sont les premières victimes. On imagine mal aujourd'hui la sévérité de cette crise. Classes moyennes et ouvrières souffriront jusqu'à la fin du siècle, et favoriseront, dans leur colère et leur sentiment d'injustice, le développement d'un socialisme américain virulent :

En 1893, plus de 600 institutions bancaires se sont effondrées. (...) En juin 1894, au moins 194 sociétés de chemin de fer avaient fait faillite (...) dont le Northern Pacific and l'Union Pacific. (...). La production du fer et du charbon diminua et pour aggraver encore les choses, la récolte de maïs fut mauvaise en 1894, tandis que les besoins de l'Europe en blé ne furent pas importants cette année-là. Chômage, grèves, mécontentement et souffrances caractérisent les hivers 1893 et 1894 . C'est l'époque de la grève des employés de Pullman à Chicago et de la marche de l'« armée de Coxey » pour demander des secours et du travail pour les chômeurs. (Nouailhat Y.H., $1982: 146$ )

La grève de Pullman sera sévèrement réprimée, tandis que seront piétinées les libertés les plus élémentaires du chômeur sans logis, qui par sa seule existence embarrassée accuse l'Amérique en silence: le vagabondage, dans les années 1890, est passible de prison. Pendant ce temps, le marasme s'étend : la production de biens durables chute de plus d'un tiers de 1892 à 1896.

31 La peur de l'immobile, la peur d'un sommeil insouciant qui verrait l'histoire passer son chemin et la terre se dérober sous les assauts de concurrents avides, a entrâné l'Amérique dans une frénésie "faustienne», et cette frénésie a paradoxalement précipité la crise et la sclérose. La Frontière, ce débouché naturel des sans-emplois, ne s'en est que plus vite refermée, laissant l'Amérique face à elle-même et au monde. Sans 
prendre le temps de l'introspection salutaire, elle préfèrera encore, pour s'extirper de la sclérose et du marais d'un espace fermé, l'élan missionnaire, en direction d'un " outre-mer» qu'on aime à décrire comme étant gaspillé entre des mains inaptes, rongé par l'injustice, voire indigne de l'Homme; car il y a l'Homme et il y a l'Autre, l'indigène des mers chaudes, par exemple, dont on prétend corriger ou éradiquer les imperfections afin de rendre le monde plus conforme à la haute idée que l'Amérique se fait de l'humanité. Le sénateur Albert Beveridge sera le chantre de cette ferveur patriotique et racialiste qui, sous la plume du révérend Josiah Strong, avait déjà pris de redoutables accents mystiques.

La politique étrangère des États-Unis à cette époque sera donc d'un enthousiasme belliqueux, et comme la quête coloniale des Européens, elle se perd dans un brouillard mythologique où guette la maladie identitaire, le mépris des races indigènes, certes, mais aussi la peur de concurrents européens atteints de la même maladie et de la même boulimie, enclins à obscurcir son timide soleil et à dévorer ses racines encore incertaines.

Concrètement, l'annexion de Hawaï, rejetée par le président Cleveland après la révolution d'opérette orchestrée par les colons américains, est approuvée par son successeur McKinley. Puis une guerre-éclair contre l'Espagne permet aux États-Unis de mettre la main sur Porto Rico, Guam, les Philippines et Cuba. Cette guerre rappelle, par son prétexte, par sa brutalité enthousiaste, mais aussi par ce mélange de naïveté et de mauvaise foi messianique, la guerre contre le Mexique. Néanmoins, c'est aussi, symboliquement, l'Europe tyrannique et colonialiste qu'on attaque à travers elle, c'est à dire un ensemble composite qui, croit-on ou veut-on croire, s'entend en coulisses contre les États-Unis.

Répugnant à affronter ses propres démons, l'Amérique est, encore une fois, allée tuer ceux des autres, transfigurant l'agression en acte libérateur - elle préfèrera d'ailleurs toujours l'interventionnisme commercial à la mainmise politique, en amont ou en aval de ses opérations militaires.

Au-delà du dérivatif aux frustrations sociales que semble traduire la guerre, nous voyons aussi cette dernière comme le refus de renoncer à un rapport au monde qui relève de la boulimie existentielle - produire, commercer, consommer et consumer afin d'exister : incapables de digérer leur propre production, les États-Unis à la veille du $\mathrm{XX}^{\text {ème }}$ siècle n'en augmentent pas moins leurs bases productives outre-mer et élargissent par la force des marchés étrangers - le marché chinois par exemple, dont ils prétendirent forcer l'accès sous prétexte d'égalité commerciale avec les grandes puissances européennes. La politique des "portes ouvertes» inaugurée par le secrétaire d'état John Hay ressemblait en fait davantage à une brutale politique de la canonnière.

\section{Ruées vers l'or : métaphores de la lutte élective et sélective}

Les coïncidences se plaisent parfois à imiter la Providence, ou les hommes à croire qu'il en est ainsi. En 1848, les États-Unis se sont emparés de la Californie, mais il leur faut la peupler afin de la rendre économiquement viable et la protéger des convoitises anglaise, russe et même vaguement française. Tâche difficile tant elle est difficile 
d'accès. Pour preuve, nombre d'immigrants (fermiers ou bûcherons) préfèrent passer par Panama et rejoindre San Francisco en bateau. Lorsque James W. Marshall découvre quelques éclats de métal brillants aux abords de la scierie où il est contremaitre du côté de Sacramento, il provoque d'abord l'embarras de son employeur. Un an plus tard pourtant, les candidats à la fortune affluent du monde entier. C'est la première fièvre mondiale de l'or, et elle permettra à la fois d'établir l'importance de la Californie comme base commerciale dirigée vers l'Asie, et de stimuler l'investissement, accélérant le redémarrage de l'économie américaine. L'or a permis de gager le crédit sur une masse métallique accrue. En résolvant provisoirement l'impossible équation entre un papier-monnaie inflationniste et une monnaie métallique (or ou argent) déflationniste, il est venu couronner la sortie de crise et le redéploiement de l'Amérique.

Lors de la crise des années 1890, c'est cette même peur de l'inflation qui convainc les Républicains de s'opposer à la frappe de l'argent aux côtés de l'or, réclamée par les milieux ruraux lors de l'élection de 1896. Elle permettrait pourtant d'augmenter la masse monétaire, et de provoquer indirectement une hausse des prix et donc des bénéfices des petits exploitants, en même temps qu'elle faciliterait l'accès de ces derniers au crédit. Or, les Républicains voient la victoire de leur candidat McKinley aux élections.

La politique monétaire de ce dernier sera intransigeante, mais, par chance, de grosses découvertes d'or l'empêcheront au même moment de trop pénaliser l'économie américaine assoiffée de crédit, à une époque où, malgré une politique étrangère agressive, l'Amérique reste économiquement et moralement déprimée. La nouvelle selon laquelle de l'or a été découvert dans le Yukon canadien, aux confins de l'Alaska, déclenche dès l'été 1897 une fièvre et une vague migratoire au départ de Seattle auxquelles seule la ruée de 1849 est comparable. Des chômeurs du monde entier s'embarquent pour l'aventure, mais aussi parfois d'honnêtes bourgeois que la crise affecte peu, sacrifiant famille et travail à leur rêve héroïque - encore davantage que l'audace du misérable, ce défi au confort révèle peut-être la profondeur de la crise, la peur de la sclérose que nous avons déjà évoquée ainsi que l'état de manque que l'or viendrait combler'. Même si la plupart des chercheurs seront déçus, voire ruinés, comme ce fut déjà le cas en 1849 , cet or va irriguer l'économie américaine et l'installer assez durablement dans la prospérité ${ }^{10}$. Ce qui nous intéresse ici, c'est la symbolique de l'or, la façon dont elle va frapper l'imaginaire populaire américain en tant que richesse trouvée et méritée, par opposition à la richesse héritée.

39 Au-delà même de leur impact économique (augmentation des réserves d'or du Trésor permettant de gager le crédit sur les réserves accumulées), elles aident l'Amérique à retrouver sa foi en elle-même:en offrant un débouché à des individus qui se retrouvent alors dans l'impasse économique, dans un pays qui doute de sa capacité à poursuivre son expansion territoriale, les ruées concourent à l'idéalisation de la Frontière comme lieu du renouveau et comme terre promise régénératrice.

C'est d'autant plus vrai que c'est à chaque fois à l'extrémité d'une Amérique en devenir que les découvertes sont réalisées. L'ouest ensoleillé d'abord, le Grand Nord ensuite, dont le surgissement au-devant des hommes de bonne volonté vient dissiper la menace d'une Amérique fermée, privée de sa Frontière bien-aimée. Certes, le Yukon est canadien, mais il s'adosse à l'Alaska nouvellement acquis, et c'est en conquérants, voire en propriétaires arrogants que beaucoup d'Américains l'abordent (pour preuve les frictions d'un Jack London querelleur avec la police montée canadienne). 
41 Le fait que ce sont des hommes simples qui découvrent l'or (Marshall en Californie, Skookum Jim, George Cormack et Dawson Charlie dans le Yukon) contribue aussi à idéaliser la Frontière comme le lieu où l'homme sans traces devient l'homme qui marque et que l'on remarque, le lieu où le mortel se fait demi-dieu. L'or lui-même, que l'on passe au crible au milieu d'un amas de cailloux sans valeur, s'impose comme métaphore de celui qui le découvre, pépite parmi les hommes, élu durement sélectionné.

Le « découvreur » est ainsi un héros de la sélection naturelle, mais aussi d'une certaine forme d'élection divine : c'est un survivant qui découvre et révèle la grâce dont il est investi en même temps qu'il découvre l'or de la mine ou du ruisseau. Il se défait de la carapace minérale de l'anonyme pour briller parmi les hommes, mais le Divin l'avait déjà reconnu dans sa simplicité rustique, et attendait de pouvoir le révéler aux hommes.

43 Cette idée, ou peut-être cette illusion, est mise au jour par Henri Miller dans sa pièce Death of a Salesman. Quoiqu'écrite après la guerre, elle puise dans la crise de 1929, qui a profondément marqué Miller et ruiné sa famille, le matériau de sa tragédie. C'est bien la crise existentielle qui attise le rêve d'or de son personnage principal, un commis voyageur endetté et vieillissant dont les employeurs ont oublié l'histoire de sueur et négligé la valeur humaine ; rêve trompeur parce qu'il est glorification du surhomme, élu parmi les hommes mais dont l'électeur serait, plutôt que Dieu, un prestidigitateur cynique. La face sombre d'une telle glorification, au bout d'un parcours humain aussi terne qu'exténuant, c'est la dépréciation, méprisante ou désespérante, de l'homme simple auquel le spectateur est pourtant invité à s'identifier.

Ce sont ainsi des milliers d'hommes simples, pour qui la vie s'efface à mesure qu'elle se vit, qui partent vers le lieu mythique de la ruée où, dit-on, les anonymes deviennent des rois. Au fond, la liberté américaine, c'est celle de devenir le roi de sa propre histoire.

Au-delà de l'imposture dont un tel rêve n'est jamais loin, la ruée révèle l'impatiente faim de vie qui crie qu'il faut se dépêcher de briller avant de s'éteindre. Spéculer plutôt qu'investir, exploiter plutôt que cultiver, se manifester plutôt qu'accomplir, voilà la face sombre de l'impatient rêve américain et le terreau de sa trahison permanente.

Il n'y aura pas d'or après 1929. Ou plutôt, l'or de cette époque, c'est le service et le sacrifice anonymes, par opposition au rêve fondamentalement égocentrique du chercheur d'or. L'Amérique des années de guerre expie l'égoïsme clinquant des années folles. Il n'est, jusqu'à présent, de crise que l'Amérique ait mieux surmontée que celle de 1929. L'introspection lente et douloureuse et le sang versé furent les conditions de ce retour au monde.

47 L'or reste, dans l'imaginaire, le seul matériau capable de mettre en mouvement et de combiner, par de subtiles alchimies, les composantes de la matière au service de celui qui le possède et qui l'exploite. Il transmet un peu de son incorruptibilité et de son éclat éternel à tout ce qu'il touche ainsi qu'à ceux qui croient s'en emparer. Il rend le frêle humain léger, brillant, lointain comme une étoile.

Aussi la faim d'Homo Americanus, qui se veut faim des hommes affranchis, est-elle teintée du désir de se soustraire, le temps d'un mensonge et d'une vie, à la corruption des corps vivants; et dire au XX ${ }^{\text {ème }}$ et au XXI ${ }^{\text {ème }}$ siècles du pétrole qu'il est l'or noir, c'est dire qu'il est la face noire du rêve aurifère de l'Amérique. 
49 L'or noir nous ramène en fait à l'orgueil et aux impostures du rêve américain. Le pétrole est lui-même corruption végétale et animale, pourriture des siècles, sombre souillure en attente de combustion et de disparition immédiates. Comme l'or, et pas seulement du fait de sa charge symbolique, il met le monde en mouvement, mais il annonce aussi sa grande panne. Tandis que l'or est quantité constante, le pétrole est quantité diminuante qui ramène la vie physique à ce qu'elle est : diminution constante de sa propre étendue temporelle.

50 Sa combustion rend possible un mode de vie extraverti peu favorable à l'introspection, comblant par la vitesse et par le bruit le vide d'une vie sans traces (l'Américain naît d'un arrachement, qu'il préfèrera toujours au pourrissement européen, c'est à dire à l'emprise de l'histoire).

51 La peur de l'introspection fut illustrée par les réactions aux attaques du 11 septembre que plusieurs membres du gouvernement américain assimilèrent immédiatement, et à notre avis de façon incongrue, à une tentative d'anéantir un mode de vie, quand c'est la présence (jugée envahissante, spirituellement néfaste, etc) des États-Unis au Moyen Orient et plus généralement dans le monde musulman qui était visée. Autrement dit, la seule mention d'agresseurs arabes fit, peut-être inconsciemment, surgir le spectre d'une interruption des livraisons de pétrole, sans lesquelles la débauche énergétique indissociable du mode de vie américain cesserait aussitôt dans les affres de l'effondrement économique - toujours la peur de l'immobile. Cette invocation obsessionnelle du mode de vie nous paraît relever de la vénération inquiète d'une abondance pourtant mal partagée, et d'une frénétique mais rassurante activité digestive :

Ces terroristes tuent non seulement pour mettre fin à des vies, mais aussi pour mettre à mal et mettre à mort notre mode de vie ${ }^{11}$ (...) Ils espèrent que l'Amérique deviendra peureuse, se retirant du monde et abandonnant ses amis. Mais la seule manière de combattre le terrorisme en tant que menace à l'encontre de notre mode de vie ${ }^{12}$ est de l'arrêter, de l'éliminer et de le détruire partout où il prospère. (Bush G. W., 20 Septembre 2001 ; traduction de l'auteur)

Il est ici question de liberté, mais d'une liberté réduite au droit à la boulimie qui évite de regarder en soi. D'où l'obsession du pétrole qui est aussi noir et poisseux que l'or est pur et brillant. On veut le pétrole et la flamme qui permettent à l'Amérique et plus généralement à l'Occident de s'agiter, de dévorer, de s'enivrer de l'illusion fugitive d'un éclat immortel. L'Amérique des années 2000 s'est peut-être déployée violemment vers l'ennemi désigné pour éviter de regarder en face l'angoisse de son déracinement. Son agressivité est la réponse d'un boulimique qui réagit à l'effondrement de sa maison en s'efforçant de sauver le frigidaire des décombres. La nouvelle ère sera peut-être celle de l'introspection et du discernement.

\section{Conclusion}

53 Les crises américaines sont peut-être des crises de l'impatience. On investit trop massivement et trop rapidement dans un territoire que le pays n'a pas encore eu le temps de digérer, dans des prêts à des consommateurs qui n'ont pas eu le loisir de devenir adultes, dans un rêve qui n'a pas encore pris forme. L'effondrement est toujours à la mesure de l'euphorie spéculative. 

est pourtant rarement pris, car s'arrêter en chemin, c'est prendre le risque de ne plus repartir, de se voir happer par un monde qui a continué à bouger et à grandir. Regarder en soi, c'est prendre le risque d'y voir la folie de l'histoire que l'on croyait avoir laissée en Europe, de l'autre côté de l'Atlantique. De plus, l'Américain a toujours trouvé son salut et l'énergie de sa renaissance dans le déploiement vers l'extérieur l'introspection culpabilisante est européenne. L'Américain agit sur son environnement, et choisit de l'étendre dans un redéploiement qui, croit-il toujours, est susceptible de résoudre ses crises économiques et morales. Ce mouvement, qui ne manque pas d'audace créative, est aussi porteur d'une violence faite à la terre et aux hommes. La faim de terres, la faim de biens et l'agitation que cela implique ne sont peut-être plus compatibles avec un monde en voie de rétrécissement.

plus difficile à digérer - c'est que la page de l'individualisme compétitif comme moteur
de la croissance et garant de la prospérité est en train de se tourner.

\section{BIBLIOGRAPHIE}

Aliber, Robert Z., Kindleberger, Charles P., Manias, Panics and Crashes : A History of Financial Crises, Hoboken, John Wiley \& Sons, 2005.

Auster, Paul, Moon Palace, London, Faber \& Faber, 1989.

Beveridge, Albert, J., " The March of the American Flag », 16 septembre 1898, in Joseph R. Conlin, History of the United States to 1877, San Diego, Coronado Publishers, 1986.

Bush, George, W., « Address to a Joint Session of Congress Following 9/11 Attacks », American Rhetoric, 20 Septembre 2001. Disponible en ligne : http://www.americanrhetoric.com/speeches/ gwbush911jointsessionspeech.htm. Page consultée le 3 juin 2013.

Clough, Shepard B., Histoire économique des États-Unis depuis la guerre de sécession, Paris, Presses Universitaires de France, 1953.

Conlin, Joseph R., History of the United States to 1877, San Diego, Coronado Publishers, 1986.

Ferguson, Niall, The Ascent of Money : A Financial History of the World, London, Penguin, 2008.

Fox, Justin, The Myth of the Rational Market : A History of Risk, Reward, and Delusion on Wall Street, New York, Harpercollins, 2009.

Campbell, Colin J., The Coming Oil Crisis, Brentwood, Multiscience Publishing Company and Petroconsultants, 2004.

Chester, Edward W., United States Oil Policy and Diplomacy : An Overview, Santa Barbara, Greenwood Press, 1983.

Gervais, Pierre, Les États-Unis de 1860 à nos jours, Paris, Hachette supérieur, 2005. 
Irving, Washington, « Rip Van Winkle », in The Sketch Book of Geoffrey Crayon, New York, CS Van Winkle, 1819.

James, Harold, The Creation and Destruction of Value : The Globalization Cycle, Harvard, Harvard University Press, 2009.

Krasner, Stephen D., Defending the National Interest : Raw materials Investments and the US Foreign Policy, Princeton, Princeton University Press, 1978.

Mélandri, Pierre, Histoire des États-Unis contemporains, Paris, André Versaille, 2008.

Miller, Arthur, Death of a Salesman, New York, Viking Press, 1949.

Nouailhat, Yves-Henri, Évolution économique des États-Unis du milieu du XIX ${ }^{\text {ème }}$ siècle à 1914, Paris, Société d'Édition d'Enseignement Supérieur, novembre 1982.

O'Brien, Patrick, The New Economic History of the Railways, Londres, Croom Helm Limited, 1977.

O'Sullivan, John, « Annexation », United States Magazine and Democratic Review (New York), vol. 17, $n^{\circ} 3$, Juillet-Août 1845, p. 5-10.

Strong, Josiah, Our Country : Its Possible Future and Its Present Crisis, New York, Baker and Taylor Company, 1891.

Turner, Frederick, Jackson, The Frontier in American History, Tucson, University of Arizona Press, 1986.

Zinn, Howard, Une histoire populaire des États-Unis, Marseille, Agone, 2002 [Édition originale : Zinn, Howard, A People's History of the United States, New York, HarperCollins, 1980].

\section{NOTES}

1. Voir mes italiques. Notez la trace du radical kreï, indiquant deux versants, ou deux états possibles du monde.

2. Idem. On retrouve encore la trace du radical kreï.

3. Le pionnier progresse vers sa propre marge, s'éloigne de son Moi social pour être plus près de sa vérité personnelle ou plus près de Dieu.

4. Voir l'épigraphe d'Auster (1989) : « Nothing can astound an American ». Notons que la citation est tirée du roman de Jules Verne De la terre à la lune, publié en 1865 et exaltant l'exploit technique qui est aussi un exploit de la volonté.

5. Rappelons d'ailleurs que c'est une Amérique esclavagiste qui guerroie au Texas, et que l'un des premiers gestes du gouvernement du Texas indépendant sera d'y restaurer l'esclavage.

6. Voir mes italiques. On voit bien par ce terme que c'est la persistance d'une crise, ou en tout cas sa perception, qui pousse une partie de l'opinion publique américaine à réclamer cette guerre contre le Mexique.

7. Voir Zinn (2002: 176). Notons que c'est en grande partie le développement du commerce avec la Chine, notamment sous l'impulsion de l'émissaire Caleb Cushing et de ses canonnières, qui permet aux États-Unis de prendre pied sur la côte Pacifique, et d'y défier non seulement le Mexique, mais encore la Grande Bretagne. Il permet tout à coup d'écouler les fourrures de l'Oregon, voire des Rocheuses, alors que le marché européen n'est plus ce qu'il était.

8. Célèbre personnage de fiction dû à l'imagination de Washington Irving, Rip Van Winkle, le dormeur que la révolution surprend au réveil d'un sommeil de vingt ans, est aujourd'hui une icône de la littérature, et peut-être de la psyche américaine. Voir Irving (1819). 
9. La fermeture de la Frontière en 1890 n'a pas manqué d'inquiéter, et le Grand Nord sera cette nouvelle frontière qu'on attendait. Plus généralement, le Frontière refermée invite à redéfinir ce terme ainsi que les promesses qu'il recèle (Frederick Jackson Turner s'y attelle, mais cette redéfinition prendra du temps et n'est toujours pas complète). Le pays de l'expansion constante se retrouve au pied du mur. Le pays de l'exploitant indépendant s'industrialise et se prolétarise. L'enivrement de l'espace sans cesse renouvelé fait place à une claustrophobie existentielle, souvent représentée par l'homme enchaîné à la machine, et que seule la littérature a pu vraiment rendre sensible.

10. La loi qui institue officiellement et solidement l'étalon-or (Currency Act) passe sans difficulté en 1900, comme pour signifier que le nouveau siècle sera américain, et que l'Amérique fondera sa prospérité sur l'or.

11. Voir mes italiques.

12. Idem. On remarquera la répétition machinale de l'expression "mode de vie" dans le même discours et à quelques secondes d'intervalle. Elle reflète le décalage entre un évènement encore largement inexpliqué au moment du discours et les certitudes auxquelles se cramponne déjà George Bush.

\section{RÉSUMÉS}

Si les crises qui ont frappé les États-Unis depuis la première moitié du XIX ${ }^{\mathrm{ème}}$ siècle ont souvent été plus brutales qu'ailleurs, elles ont aussi été davantage porteuses de changements tangibles et de promesses irrationnelles. Ces fausses apocalypses, quoiqu'involontairement fécondes, sont pourtant vite oubliées.

Il n'est pas absurde d'interpréter chaque nouvelle crise comme résultant de l'abandon temporaire du territoire américain à la convoitise de quelques hommes, à mesure que le désir originel de la psyche américaine (désir de renouvellement spirituel, ou tout au moins moral) tend à s'affaiblir.

Ce schéma de «crise par voracité » peut s'appliquer aussi bien à la crise des années 1830 qu'à celle des années 1890 . Nous verrons s'il en est de même pour celle qui affecte aujourd'hui la plupart des pays occidentaux.

Crises in the US since the first half of the nineteenth century have often been more brutal than elsewhere, but have also been pregnant with more tangible changes and irrational promises. These would-be apocalypses, although unwittingly fertile, are also fast forgotten.

Each new crisis tends to be interpreted as stemming from the subjection of America's territory to a few men's impatient craving for possession, while the original yearning shaping the American psyche - for spiritual, or at least moral, regeneration - gradually fades into oblivion.

This desire-generated crisis pattern seems to match the crises of the 1830 s and the $1890 \mathrm{~s}$. To what extent it fits in with the current crisis is what we shall also try to determine.

Las crisis que han afectado a Estados Unidos desde la primera mitad del siglo XIX, no sólo han sido más brutales que en otros paises, sino tambien preñadas de cambios tangibles y de promesas irracionales. Estas falsas apocalipsis, aunque rápidamente se olvidan, han ido fertilizando y estimulando a la sociedad americana. Hay los que interpretan cada nueva crisis como procedente de que el espacio físico de América sea sometido al ansia irreprimible de explotación, mientras 
que el deseo de regeneración espiritual, o al menos moral, que aquel espacio debía satisfacer al colonizarse América del Norte, disminuye y se olvida. Tal fenómeno se produjo, o más bien se percibió, en los años 1830, y otra vez en los años 1890 . Veremos en qué medida estas crisis se parecen a la que nos está afectando ahora.

As crises que afetaram os Estados Unidos desde a primeira metade do século XIX foram freqüentemente mais ásperas que em outro lugar, mas também mais portadoras de mudanças tangíveis e promessas irracionais. Esses falsos apocalipses, mesmo se involuntariamente fértieis, são rapidamente esqueçidos. Não é absurdo interpretar cada nova crise como resultando do abandono temporário do território americano na ganância de alguns, na medida que o desejo original da psique americana (desejo de renovação espiritual, ou pelo menos moral) tende a debilitar. Este plano de «crise por ganância » pode aplicar-se tanto na crise dos 1830s como aquela dos 1890s. Nós veremos si é o mesmo para a crise que afeta a maioria dos países occidentais hoje.

\section{INDEX}

Palavras-chave : história, crise, banco, conquista, fronteira, descoberta, ouro

Mots-clés : histoire, crise, banque, conquête, frontière, découverte, or

Keywords : history, crisis, bank, conquest, frontier, discovery, gold

Palabras claves : historia, crisis, banco, conquista, frontera, descubierta, oro

\section{AUTEUR}

\section{JEAN LUC TENDIL}

Jean-Luc Tendil est professeur agrégé au département LLCE anglais de l'université d' Avignon, où il enseigne la civilisation et l'histoire des États-Unis, la littérature américaine, la traduction et la grammaire depuis 2004 .

Auteur d'une thèse sur Jack London soutenue à Montpellier en 2007 à l'université de Montpellier sous la direction de M. Bandry, il y analyse l'influence sur la littérature des États-Unis, et en particulier sur l'oeuvre de Jack London, de l'idéologie évolutionniste et de l'expansionnisme américains (De la conquête territoriale à la conquête sociale, l'homme jouet ou acteur de son évolution dans l'oeuvre de Jack London?).

Ses travaux ont d'abord concerné différents aspects de l'oeuvre de London (préface de To Build a Fire, recueil de nouvelles publiées chez Zulma en 2006 ; communication et publication pour le laboratoire ICTT à l'université d'Avignon en 2005).

Ses activités d'enseignement l'ont aussi amené à s'intéresser à la littérature française rurale du

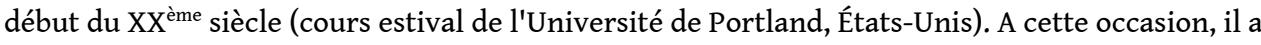
également animé une conférence sur l'héroïsme marginal et l'héroïsme fortuit dans le cinéma français des années 50-60.

Il a participé en novembre 2009 au Congrès de l'Institut des Amériques dédié à la crise aux ÉtatsUnis. Sa communication s'y intitulait : « Les crises aux États-Unis depuis 1837 : facteurs de renouveau et de redéploiement $»$.

Ses travaux les plus récents concernent la traduction commentée d'un poème narratif de Robert Service, travail présenté à l'Université de Mostaganem (Algérie) en novembre 2010, et un essai sur les rapports entre l'écrivain Jack London et l'argent, dans le cadre d'un ouvrage collectif dirigé par Hervé Larissa en 2012, Les écrivains et l'argent. 